\title{
PID - GAIN SCHEDULING CONTROLLER FOR A ROBOT MANIPULATOR
}

\author{
Carlos Pérez, Carlos Vivas, F. R. Rubio
}

\author{
Dept. Ingeniería de Sistemas y Automática \\ Escuela Superior de Ingenieros. \\ Universidad de Sevilla. Camino de los Descubrimientos $s / n$. \\ 41092-Sevilla. SPAIN \\ Telf. +349544873 50, Fax: +34954487340 \\ e-mail: $\{$ cpf, vivas, rubio\}@cartuja.us.es
}

\begin{abstract}
This paper presents the implementation of an adaptive PD - Gain Scheduling controller for a six-degree-of-freedom robot manipulator. Uncoupled PD controllers are proposed for each joint where each single manipulator joint is controlled independently of the others. The gain scheduling technique enables the controller to adapt on-line the PD parameters depending on the operation conditions (essentially the robot arm position). The nonlinear dynamic model of the robot arm is then linearized discarding non-linear terms which are considered as model uncertainties, and a continuous adaptation law for the PD controller parameters is proposed. The overall control strategy can be regarded as composed by a non-linear transformation (adaptation law), followed by a linear PD regulator. This simple technique offers promising results with respect to the traditional worst-case PD design.

Copyright $\odot 2000$ IFAC
\end{abstract}

Keywords: Robotics; PID Tuning; Gain Scheduling; Adaptive Control

\section{INTRODUCTION}

The rapidly growing interdisciplinary field of robotics, where systems must operate satisfactorily with different levels of imprecise information, represents one area where adaptive control is finding extensive application.

The use of adaptive controllers requires very little justification in robot manipulators. A variety of factors including parametric and structural uncertainties, and nonlinearities that usually are neglected in the modeling process, make adaptive control mandatory. In addition a variety of disturbances are also present, and accurate trajectory tracking may become a hard goal to meet.

Parametric uncertainties are usually present due to imprecise knowledge of the manipulator mass properties, unknown loads, or uncertainty in the load position in the end-effector. Structural uncertainties arise from the presence of high-frequency unmodeled dynamics, actuator dynamics, flexibilities in the links or joint plays. In the presence of such uncertainties, linear feedback controllers can not provide good performance and, hence, adaptive solutions are required.

If Gain Scheduling is to be used, it is usually necessary to have a good insight into the dynamics of the process, in order to simplify the controller design. Thus, gain scheduling seems to be a promising and simple control strategy for a robot manipulator, since the nonlinear dynamics of these systems are well known and modeled. According to this, following is presented a PD - Gain Scheduling adaptive controller for a six degrees of freedom robot arm manipulator. 
This paper is organized as follows: In section 2, a description of the robot arm manipulator employed is given, as well as some aspects of the robot dynamic model. Section 3 is dedicated to present the adopted control structure. Section 4 presents some simulation results with the proposed controller and finally, conclusions are given in section 6 .

\section{SYSTEM DESCRIPTION}

The Gain Scheduling controller proposed in this work has been designed for the RM10 Robot Manipulator. The RM10, shown in figure 1, is a six-degree-of-freedom revolute joint manipulator arm.

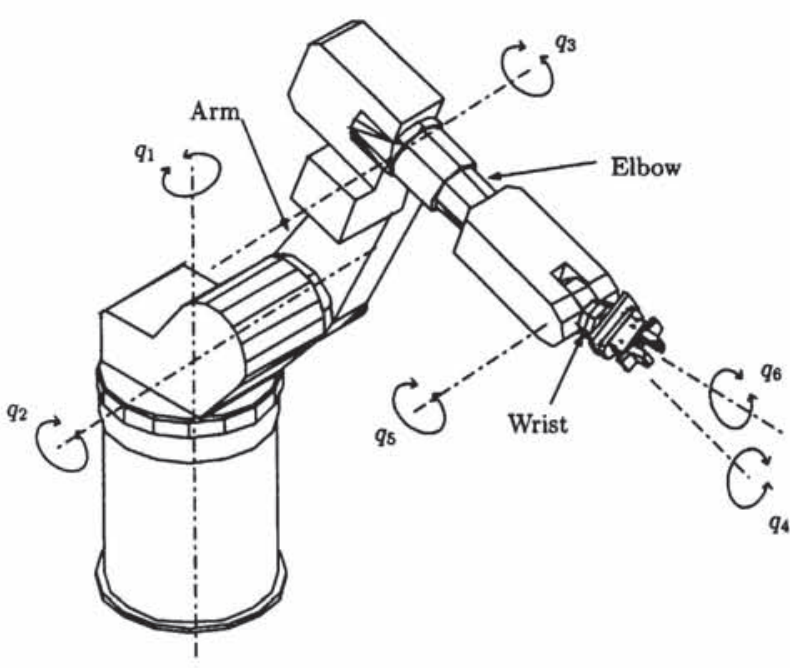

Fig. 1. The RM10 Robot Manipulator

All the six joints are driven by DC-brushless lowinertia electric motors which provide an uniform torque for all joint positions, and enables high control torque peaks. Torque is delivered to the joint axis through gear reductions, thus RM10 is an indirect-drive manipulator. The joints also provide an electric brake to block the manipulator arm in any position, (Robótica Industriale, 1991).

Coupled to every motor axis is a two-pole resolver device which provides accurate measure of the correspondent joint position. These measures will permit, as usual, the closed-loop control of the system. The RM10 system employs an VME bus based architecture, providing independent control boards for every joint. Particularly, the real-time DS1103 control board, dSPACE trade, was employed (dSPACE, 1998), (MOOG GmbH, 1990), (dSPACE, 1999). The control board were plugged into the expansion bus of commercial PC, holding a $333 \mathrm{MHz}$ PowerPC as main processor and an additional DSP as input/output processor.

The design and simulations of controllers in this paper has been supported by the popular
SIMULINK 2.0 toolbox under MATLAB 5.2 environment. For the implementation of control algorithms the dSPACE Real Time Workshop Toolbox has been also used.

Before accomplishing the design of a controller it is necessary to obtain a dynamic model of the robot manipulator. Following the Euler-Lagrange formulation, (Craig, 1989), the dynamic model of a general n-link rigid-body robot is a second order nonlinear equation:

$$
J(q) \ddot{q}+C(q, \dot{q}) \dot{q}+F(q, \dot{q})+G(q)=\tau
$$

where $q \in \mathcal{R}^{n}$ is the joint position; $\tau$ is the generalized torque; $J(q)$ (Inertial forces), $C(q, \dot{q}) \in \mathcal{R}^{n x n}$ (Coriolis and centripetal forces terms), $F(q, \dot{q}) \in$ $\mathcal{R}^{n x n}$ (Friction model) and $G(q)$ (gravitational components) $\in \mathcal{R}^{n}$ are nonlinear functions of $q$ and $\dot{q}$.

The equation of motion is complex and contains a number of hard to handle nonlinear terms. In order to simplify the controller design, Coriolis, centripetal and gravitational terms in equation 1 have been neglected, and included as model uncertainty.

A number of additional parameters are required to characterize the dynamic model of a robot manipulator, such as link masses and inertias. These parameters have been estimated by geometric measures and dynamic experiments of the robot arm.

\section{CONTROL STRUCTURE}

In this section is described the structure of an adaptive PD-Gain Scheduling controller for the previously described RM10 robot arm manipulator.

Several ways of dealing with the non-linear control problem in robot manipulators are proposed in classical literature. One of the most simple approaches consists of linearizing the dynamic model equations of the robot arm, neglecting the coupling between links. In this case, each robot axis is treated as a SISO system that can be modeled as, (Sciavicco and Siciliano, 1996)

$$
m_{i} \ddot{q}_{i}+b_{i} \dot{q}_{i}=\tau_{i}-d_{i}
$$

where $q_{i}$ is the angular displacement of joint $i$, $\tau_{i}$ is the actuator torque and $d_{i}$ is a disturbance due to Coriollis and centripetal acceleration, gravitational load and Coulomb friction Torque. This assumption is done frequently in industrial robots with gearing between the motor and the link. The problem becomes then to control a number 
of second order plants in the presence of disturbances. This kind of controllers are usually designed employing the worst-case rule, so that the performance of the system is not very precise for operation points far from the linearization point.

A second category of controllers includes the algorithms based on what is commonly referred to as the computed torque method. The basic idea is to determine the terms $J, C, F$ and $G$ in equation 1 at every instant of time, and cancel the nonlinear terms thereby decoupling the plant. Control is then effected by generating a control torque that results in a linear time-invariant homogeneous error equation whose equilibrium state is asymptotically stable.

One of the main drawbacks of this method is the necessity of computing the terms of equation 1 at every instant of time. This computation may be quite time-consuming, depending on the configuration and complexity of the system to be controlled, and may happen that the controller can not meet the computation time requirements. Most of the solutions proposed for this problem consist of considering some specific terms as a constant, so that computations can be accomplished meeting the required times.

A different approach to this problem is proposed in this paper: To avoid the former requirement of an on-line computation of the non-linear terms in equation 1 , no feedback linearization is accomplished as in the computed torque method. Nonlinear terms are instead included as uncertainties in the manipulator model and the system is controlled as six-order uncoupled system. This way equation 2 holds for every joint.

In order to compensate the neglected non-linear dynamics, an adaptive gain scheduling approach is proposed for the controller design, (Aström and Wittenmark, 1989). Thus, the nonlinear system dynamic is compensated by means of a non-linear adaptation law (see figure 2) which regulates the parameters of the PD controllers according to the operation conditions of the plant (robot link positions). The regulator thus obtained can be regarded as composed by a nonlinear transformation (adaptation law), followed by a linear PD regulator.

A main problem in the design of systems with gain scheduling is to find suitable scheduling variables. As far as robot manipulator control concerns, the variables which determine the robot operating condition correspond to the joint angular displacements $q$, and its derivatives $\dot{q}$. For the problem of concern, a six-degrees-of-freedom robot manipulator, this amounts to 12 parameters. Fortunately, the robot operating condition, say the value of the inertial, Gravitational and
Coriollis/Centripetal terms, depends weakly on most of these variables, and the dependency on this terms can be neglected. Thus, the Coriolis and centripetal forces terms can be neglected for moderate speeds in the whole manipulator work space, so operating condition dependence on $\dot{q}$ is discarded.

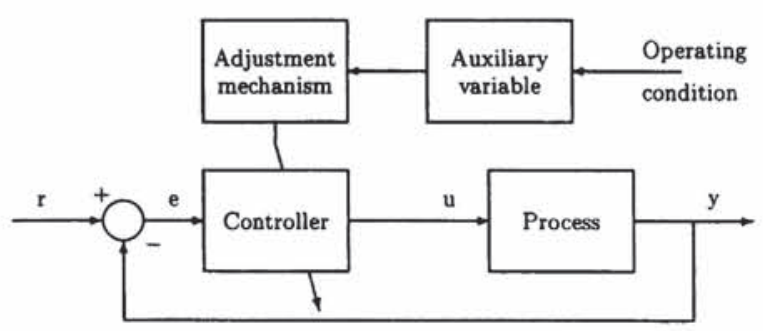

Fig. 2. Diagram of the gain scheduling structure

The most important dependencies appear in the principal diagonal components of matrix $J(q)$ in equation 1, the inertial terms. Since the control scheme do not explicitly consider couplings between links, only the diagonal elements in matrix $J(q)$ are considered to depend on the operating conditions. Furthermore, not all these terms present a significant dependence on every $q$ parameter. For the RM10 physical configuration, the main dependence in $J(q)$ is associated to the angle displacements in joints 2 and $3,\left(q_{2}\right.$ and $\left.q_{3}\right)$, and to a minor extent to the rest of joints.

\subsection{Gain Scheduling compensator design}

The gain scheduling adaptation law is designed as a continuous PD-parameter compensator. That is, the parameters of the six independent PD controllers are continuously changed in an openloop fashion depending on the robot arm position. Gain scheduling can thus be viewed as a feedback control system in which the feedback gains are adjusted using feedforward compensation.

Neglecting Coriolis/centripetal, gravitational and coulomb friction terms and linearizing equation 1 for a robot arm position, the following approximate expression holds for the system dynamics of each independent joint:

$$
\tau=J \ddot{\theta}+B \dot{\theta}+\tau_{p}
$$

where $\tau_{p}$ is a disturbance torque including the neglected dynamics.

The PD control structure is described in figure 3 and includes proportional and derivative terms with constants $K_{p}$ and $K_{d}$ respectively. The disturbance torque, $\tau_{p}$, is included as a external disturbance input, $u_{p}=\tau_{p} / K_{m}$, where $K_{m}$ is a motor actuator constant. 
The closed-loop dynamic from the desired joint position, $\theta_{d}$, to the robot effective joint position $\theta$ is described by:

$$
\frac{\theta}{\theta_{d}}=\frac{K_{p} K_{m}}{J s^{2}+\left(K_{m} K_{d}+B\right) s+K_{p} K_{m}}
$$

The controller parameters, $K_{d}$ and $K_{p}$, are computed by forcing the dynamic in equation 4 to a critically damped second order response. Thus, assuming such response as:

$$
p(s)=s^{2}+2 \delta \omega_{n} s+\omega_{n}^{2}
$$

and identifying terms in both equations, 4 and 5, the following identities hold

$$
\omega_{n}=\sqrt{\frac{K_{m} K_{p}}{J}} ; \quad \delta=\frac{B+K_{m} K_{d}}{2 \sqrt{J K_{m} K_{p}}}
$$

From the previous expressions is easily observed that as $J$ decreases, the natural frequency response of the system, $\omega_{n}$, increases yielding faster responses. On the other hand, this faster dynamic is counteracted by increasing values of $\delta$ as $J$ decreases, yielding overdamped responses. These factors counteract each other in the case of constant parameters controller (classical PD control) inducing system stability for a wide range of operating conditions.

In order to improve performance of the system with respect to a classical PD controller, a simple adaptation law for the gain scheduling controller is proposed. The proportional gain parameter, $K_{p}$, is fixed for all operating conditions while the derivative parameter, $K_{d}$, is adjusted to get a critically damped second order response according to equation 6 , that is $\delta=1$.

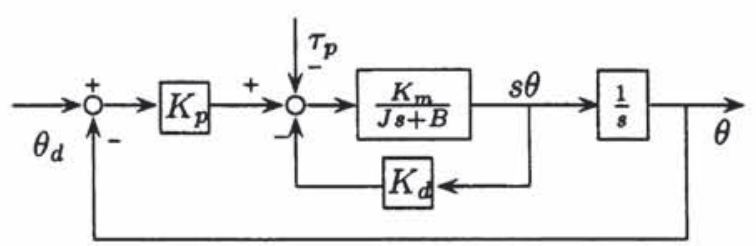

Fig. 3. PD Control Structure

In order to guarantee a critically damped close loop response a PD controller are designed as:

$$
K_{p}=\frac{J \omega_{n}^{2}}{K_{m}} ; \quad K_{d}=\frac{2 J \omega_{n}-B}{K_{m}}
$$

The matrix $J$ is calculated in every position of the manipulator, so that, the position is scheduling variable in the adaptive controller.

\section{SIMULATION RESULTS}

The Gain Scheduling design was examined by computer simulation and compared with fixed gain linear controller. The simulation uses an arm model of the RM10 manipulator (Fig. 1). The sim-

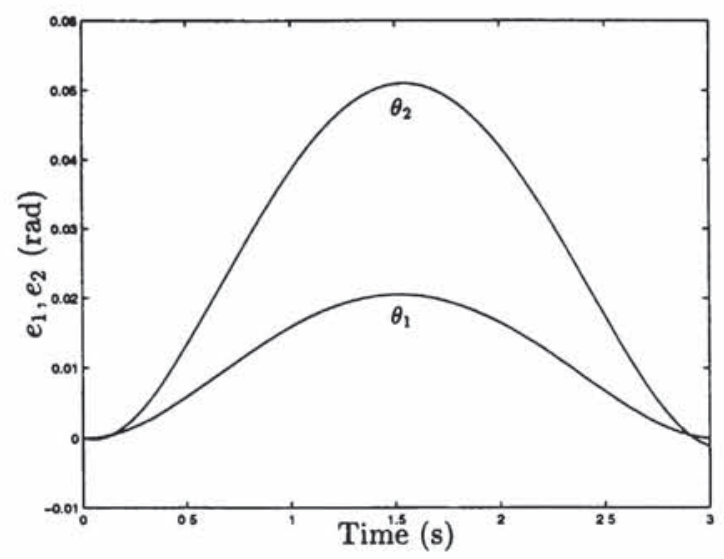

Fig. 4. Trajectory error with PD controller

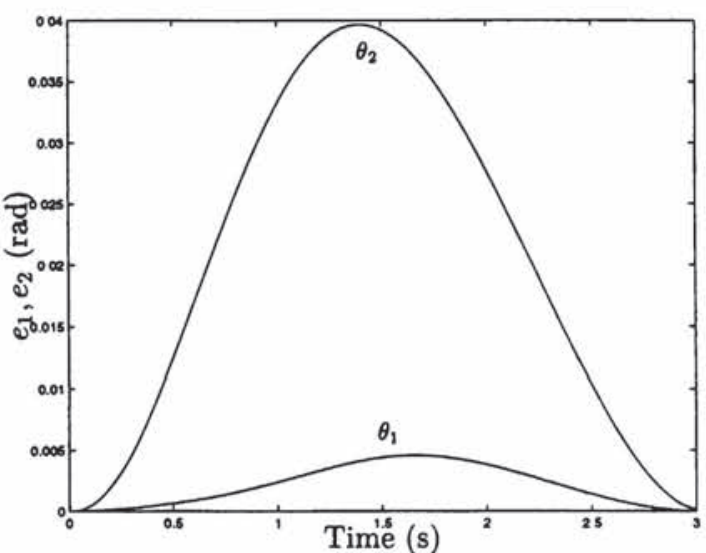

Fig. 5. Trajectory error with Gain Scheduling controller

ulation verifies how a PD controller was improved if gain scheduling is applied for $K_{d}$ gain, especially in trajectories with high inertia variation for axes 1 and 2. For a critically damped response an adaptation law for $K_{d}$ gain is used along the trajectories. Figures 4 y 5 shows tracking errors for axes 1 and 2 when a continuous adaptation law was implemented.

It can be observed, that for trajectories moving from high inertia to low inertia configuration, a classical PD controller works with acceptable results, because the increasing damping factor which would yield slower response, is compensated by an increasing $\omega_{n}$. Thus, a $K_{d}$ scheduling improve the system performance.

\section{CONCLUSIONS}

An adaptive PD gain scheduling controller is proposed for the six-degree-of-freedom robot manipu- 
lator RM10. The controller avoids computational expensive feedback linearization schemes, as the classical computed torque method, considering instead an uncoupled system model. Neglected model couplings and non-linearities are compensated by means of a continuous adaptation law which tunes the PD controller according to the manipulator operating conditions. This control strategy yields promising results in terms of trajectory error with respect to the classical fixed parameters PD controllers.

Acknowledgments. The authors would like to acknowledge CICYT for funding this work under grant TAP98-0540.

\section{REFERENCES}

Aström, K.J. and B. Wittenmark (1989). Adaptive control. Addison Wesley.

Craig, J.J. (1989). Introduction to robotics, mechanics and control. Addison Wesley.

dSPACE (1998). DS1103 PPC Controller Board. Instalation and Configuration Guide.

dSPACE (1999). Real Time Interface (RTI and RT-MP) Implementation Guide.

MOOG GmbH (1990). T158-11X Controller User Manual.

Robótica Industriale (1991). RM10 Manuale d'uso. System Robot.

Sciavicco, L. and B. Siciliano (1996). Modelling and control of robot manipulators. Mc Graw Hill. 